\title{
CONDITIONAL CONSTRUCTIONS IN NON-PROCEDURAL LANGUAGES OF ROBOTIC SYSTEMS
}

\author{
Ipatov Oleg, Vasilevsky Alexander, Rudskoy Andrey \& Yamschikov Yury
}
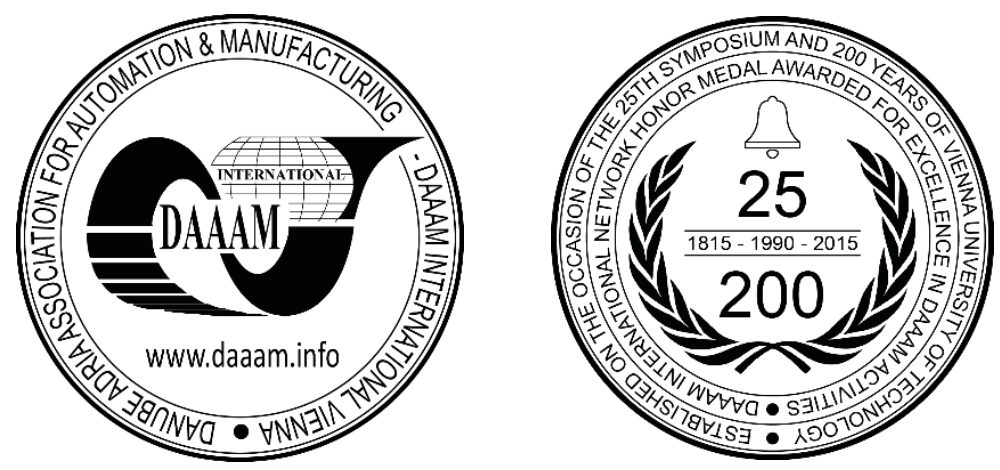

This Publication has to be referred as: Ipatov, O[leg]; Vasilevsky, A[lexander]; Rudskoy, A[ndrei] \& Yamschikov, Y [ury] (2016). Conditional Constructions in Non-Procedural Languages of Robotic Systems, Proceedings of the 27th DAAAM International Symposium, pp.0463-0466, B. Katalinic (Ed.), Published by DAAAM International, ISBN 9783-902734-08-2, ISSN 1726-9679, Vienna, Austria DOI: $10.2507 / 27$ th.daaam.proceedings.069

\begin{abstract}
The article researched and developed a universal theoretical concept of conditional constructions in non-procedural languages of different robotic systems. A multivariate classification of conditional constructions of non-procedural languages of robotic systems is offered. It is shown that the presented concept allows conditional constructions to be described in a form which allows to present them explicitly as they are common, especially their semantic properties and their typological primarily formal differences. A well-known thesis is discussed in the paper. It says that the conditional non-procedural design language fairly accurately reflects the logical operation of implication, and the notion of material implication is the source for the semantic definition of conditional constructions. A methodological basis of semantic research, pragmatic and communication properties of conditionals in the non-procedural languages of robotic systems is offered. A distinction of conditional constructions in non-procedural languages robotic systems into even and odd patterns is also researched.
\end{abstract}

Keywords: conditional constructions; non-procedural languages; robotic systems; cognitive processes; knowledge; judgment; methodology; concept; analysis.

\section{Introduction}

The interest to the problem of conditional constructions in robotic systems can be explained by the following circumstances.

At first. Taking into account the attention to conditional constructions, it is possible to conclude that they are still not enough studied from the universal typological position [6]. There are no any studies based on a correct theoretical information providing a detailed description of the conditional constructions in non-procedural languages of the robotic systems of different structures.

Secondly. Until now the initial setting of research was a well-known thesis that the conditional constructions reflect the logical operation of implication quite accurately. The notion of the material implication is the reference for the semantic definition of conditional constructions. The preliminary results show that the reliance on the logical tradition in the description and interpretation of conventional constructions requires substantial correction [2]. 
The implicative judgments tested by the logic, constitute only a part of structures that are called conventional. The content of the propositions in the statement as well as the presence / absence of the meaningful communication between these propositions is irrelevant for the logic [7]. The robotic system requires a semantic relationship between propositions, denoted in the statements. To establish how to use implicative judgments on the basis of new knowledge, it is necessary to identify and describe the conditional constructs like an object, with its own meaning and formal specificity.

Thirdly. Until now the main object of the study were bi-predicative constructions in the field of robotic systems. Even a rigorous mathematical proof is much shorter than the reasoning of orders formalized in predicate style [12]. In this regard, it is necessary to have the effective methods of investigation in relation to bi-predicative constructions.

\section{The conditional structures in procedural languages of robotic systems}

A necessary condition for the creation of robotic systems that would be able to replace a human in hazardous conditions is developing means of intelligent control and autonomous navigation providing the possibility of making independent decisions [11]. So it is very important to develop theoretical concepts, which allow to describe the conditional constructions of non-procedural languages of robotic systems. This provides the ability to represent both their common, first and foremost, semantic properties and their typological primarily formal observed differences explicitly. It demands the creation of the multi-dimensional classification of the calculated conditional constructions and exploring their prototypical and peripheral types, as well as the development of a universal metric of the conditional constructions combining different sets of issues and their characteristics.

The robot acquires information from the environment through its sensors, which provides the input signals to the controller [14]. The understanding of the conceptual and behavioural organization of intellectual capacity to design and interpret conventional constructions ensures comprehension of cognitive processes, linguistic competence and outlet strategies of the robotic systems. There are some studies which cover the classification of prototypical contingent of relevant constructions, their formal and semantic features. The contingent constructions, which in formal terms differ from the prototypical, yet remain unremarkable for the researchers. According to the other popular studies, it should be noted that the problem of synchro-diachronic semantic and syntactic relations of the conditional constructions with other language constructions, especially with time, concessive or causal, still exist [10]. The conditional constructions, as well as the causal, concessive constructions, and the constructions of value and purpose have the total value of the conditionality. The conditional structures are closely related to the relative, interrogative, and some other structures. Formal differences between conditional structures and the others can be quite shaky, whereas the standard measures of conditional values in certain contexts express different meanings. There are also situations where the construction is in principle ambiguous. The construction can allow both the conditional and temporary reading, the difference between them is a little.

\section{Some problems of the universal typological analysis of conventional constructions}

During the universal typological analysis of conventional constructions there can appear a number of significant problems:

- It is necessary to establish whether the concept of "default value" is undetectable, or it is possible to offer a universal definition that can reliably restrict the empirical data of various non-procedural languages of the robotic systems corresponding to this concept.

- Those substantive parameters that are necessary and sufficient for the construction of a universal pragmatic expedient enumerative classification of the conditional constructions should be identified.

- It is necessary to build a calculated classification of the conditional structures and determine which of the logical possibilities provided by the calculation are implemented in the language of robotic systems. In addition, it is necessary to find the causes of the established distribution of logical possibilities.

- It is necessary to establish and explore the semantic, pragmatic and communicative features of the conventional constructions, determining the preferred use of certain calculation models in the construction of discourse.

- It is necessary to determine which formal indicators and their aggregates, are directly or indirectly used in the nonprocedural languages of robotic systems for the labeling of conventional structures and their different semantic types.

- It is necessary to determine which concepts are semantically close to the concept of "default value". It can determine the possibility of using the same formal indicators for the labeling of all semantically close concepts. As a result, it will be possible to determine the certain place of the conditional constructions in the the system of other constructions which are close to each other semantically and formally.

- There should be a metric which will be used for a description of conditional constructions in different languages of therobotic systems.

The methodological concept of solving the problems mentioned above is available in the following content.

The initial setting of semantic research, pragmatic and communicative properties of the conventional constructions is based on the well-known thesis that the conditional construction of the non-procedural language fairly accurately reflects the logical operation of implication. The notion of material implication is the source for the semantic definition of the conditional constructions. The semantics of conditionality is as close as possible to the content of logical and grammatical category of a functional compliance or a function. 
In the study of formal syntactic types of conditional constructions it is necessary:

- to determine which empirical facts oft he non-procedural languages of the robotic systems correspond to the concept of "default value" semantically;

- to analyze the formal-syntactic heterogeneity oft he proposals, including the conditional construction;

- to develop metrics for the parameters oft he classification for such proposals.

Among these parameters area formal separability / non-separability of the unextractable parts of conventional structures; the presence / absence of a formal connection between the parts of the conditional structure; a status of the indicator of the communication [4].

The conventional concept must be seen as a universal semantic primitive. All attempts to define a conditional concept containing in the canonical conditional sentences, turned out to be unsuccessful because the definitions have such conceptions as the hypothetical conclusions. The possible worlds that are more complex comparing with the conventional concept need to have the independent definition either.

It should be noted that in the logic of the concept of connection does not appear in the analysis of the implications, which appears in the non-procedural languages of the conventional structures. Some experts in the field of robotic systems proved that the table of material implication loses communication idea, which is present in the case of if-then [1]. It is necessary to examine whether the relationship between the proposition-condition and the proposition-the result is casual, random or conditional. It is necessary to prove that the concept of "notional connection" is fundamental. It can not be reduced to any other concepts as it is a conceptual entity itself.

The conventional ctructures are a complex object, possessing a variety of specific, substantive and formal features. They can be subjected to a number of independent parameters. Some of these parameters are relevant for both conventional structures and other types of structures. Some of them - only for conventional structures. The number of small variations with respect to the basic decision determines the size of the search space [13]. Therefore, each of the independent features can be considered to be a basis for the classification of conventional constructions. Thus, it is possible to offer as many classifications as classification parameters [9].

When it comes to multi-dimensional classification enumerative it is important to pay attention on the selection criteria for this classification parameters, ie, how many and which parameters should include this classification. It seems that there is only one common requirement, which must be demanded from the typologically oriented enumerative classifications. All parameters of such classifications should be semantic. This requirement requires no explanation because only semantics but not a form can be a common component of a wide variety of languages. Only after the building of the universal semantic models of the conditional constructions it is possible to move on to the question concerning the formal means of these models and how they are translated into specific non-procedural languages, and how to classify the languages, depending on what kind of formal tools they use to express the certain universal semantic models of conventional constructions.

From the systemic point of view, all the researched models are logically equivalent for its semantic features, its pragmatic preferences and limitations, and their representation in the texts. These models are noticeably different from each other. It ultimately depends on the specific combinations of the classification parameter values that define the personality of each model [3].

The most important is the distinction between the real conditional constructions (odd models) and unreal conditional constructions (even models) [5]. However, for the realization of the value of both real and unreal conditional constructions the important role is played by this or that specific conditions and time localizations (at least) of the investigation. According to this, all realized models should be divided into three groups: the first group includes those models, in which the condition is localized in the future; the second group consists of those models, in which the condition is localized in the past; the third group consists of those models, in which the condition is localized in the present. The relationships between the cause and the effect influence the representation of the models in the. [8] Thus implemented models can be divided into two groups: the first group includes models in which the condition precedes the effect; the second group consists of models in which the condition ist he same as the investigation. So it is necessary first of all to get semanticpragmatic characteristics of real conditionals.

\section{Conclusion}

The importance of researching the problem of conditional constructions in non-procedural languages of the robotic systems is clear. These constructions are the key, giving the opportunity to explore and describe the thought processes of robotic systems. Their specificity is proved by a direct reflection of the cognitive ability to reflect the alternative judgments and draw conclusions, based on incomplete and insufficient information to suggest possible correlations between situations and to understand how the world would could change if the correlations were different. 


\section{References}

[1] Kasevich VB The semantics of the conventions and convention semantics. // Proceedings of the international conference. Vol.1, M. 1995.

[2] Apresyan YD Lexical Semantics. // St. Petersburg, publishing house "Nauka", 1998.

[3] G.T. Stump. The Semantic Variability of Absolute Constructions // Reidel: Dordrecht, 2001.

[4] Fedulaev FG, Karpechenko PA, OA Nikolaev, Jirov VS Inference rules of predicate logic. St. Petersburg, publishing house SPGU 2002.

[5] Bondarenko VK, Fedulaev FG Knowledge representation rules and the conclusion. Abstracts of the second AllRussia conference "Representation and use of knowledge", p. 35, Kazan, 2003.

[6] Nikoltsev VA Korzhavin GA, Podoplekin YF, Vasilevsky AS Calculated classification conditional constructions declarative languages like. Scientific and practical journal "Information and Control Systems". Electronics industry. St. Petersburg, 2006, p. 30-32.

[7] AS Vasilevsky, Podoplekin YF, Lapshin KV Methodology problems of conditional constructions in the systems of presentation and use of knowledge. // Proceedings of the Tenth National Conference on Artificial.

[8] G.F. Luger. Artificial Intelligence: Structures and Strategies for Complex Problem-Solving (6th Edition) // Addison Wesley, 2008.

[9] Alexander Vasilevsky, Lapshin KV Multidimensional classification calculated conditional constructions in nonprocedural languages intelligent systems // Proceedings of the Twelfth National Conference on Artificial Intelligence with international participation KII 2010, Volume 1, M., FIZMATLIT 2010.

[10] VV Gorelov, Lapshin KV Research sintatiko morphological problems of conditional constructions in non-procedural languages, knowledge processing systems. // NA-tehn. Sat. FSPC JSC "Concern" Granit-Electron "," Ship and aircraft multi information management systems. " // Vol. №16, p. 11-17, 2011.

[11] Smirnova, E. Stepanov, D. \& Goryunov, V. (2016). A Technique of Natural Visual Landmarks Detection and Description for Mobile Robot Cognitive Navigation, Proceedings of the 26th DAAAM International Symposium, pp.0905-0911, B. Katalinic (Ed.), Published by DAAAM International, ISBN 978-3-902734-07-5, ISSN 1726-9679, Vienna, Austria, DOI: 10.2507/26th.daaam.proceedings.126.

[12] Kuznetsov OP Cognitive semantics and artificial intelligence // Artificial intelligence and decision-making, the Federal Research Centre "Information and Management" of the Russian Academy of Sciences, 2012. № 4. S. 3242.

[13] Diveev AI Shmalko EY Numerical synthesis of group control system of robots by symbolic regression. // Proceedings of the SFU. Engineering, number 10 (171). Pp 29-45, 2015.

[14] Nehmzow U., Akanyeti O., Billings S.A. Towards modelling complex robot training tasks through system identification // Robotics and Autonomous Systems, V. 58, №3. PP. 265-275, 2010. 